\title{
The hippo signaling pathway: implications for heart regeneration and disease
}

\author{
Dominic P Del Re
}

\begin{abstract}
Control of cell number and organ size is critical for appropriate development and tissue homeostasis. Studies in both Drosophila and mammals have established the Hippo signaling pathway as an important modulator of organ size and tumorigenesis. Upon activation, this kinase cascade modulates gene expression through the phosphorylation and inhibition of transcription co-activators that are involved in cell proliferation, differentiation, growth and apoptosis. Hippo signaling serves to limit organ size and suppress malignancies, and has been implicated in tissue regeneration following injury. These outcomes highlight the important role that Hippo signaling plays in regulating both physiologic and pathologic processes. In this review, an overview of the signaling pathway will be discussed as well as recent work that has investigated its role in cardiac development, regeneration and disease.
\end{abstract}

Keywords: Hippo signaling pathway; Heart disease; Myocardial regeneration

\section{Introduction}

Myocardial infarction (MI), or insufficient blood flow to the heart muscle, promotes thedeath and loss of cardiomyocytes resulting in heart damage and impaired cardiacfunction. While patient survival following MI has improved, the prognosis is typicallypoor and can eventually progress to heart failure, a leading cause of morbidity andmortality [1]. Because mature cardiomyocytes have a limited capacity to reenter the cellcycle and proliferate [2,3], the ability of the adult heart to regenerate is similarlyrestricted and cannot adequately replace lost cardiomyocytes. The Hippo signalingpathway is evolutionarily conserved from flies to mammals and has emerged as animportant regulator of both cell survival and proliferation $[4,5]$. Importantly, this cascadealso appears critical for proper mammalian heart development and the post-natal responseto cardiac stress and injury[6-8]. It is therefore plausible to hypothesize that Hipposignaling could be targeted to promote heart regeneration after MI and heart injury. Thisreview will provide an overview of the Hippo pathway and examine its role in cardiacdevelopment, disease and regeneration.

\footnotetext{
Correspondence: delredo@njms.rutgers.edu
}

Cardiovascular Research Institute and Department of Cell Biology and

Molecular Medicine, New Jersey Medical School, Rutgers Newark, NJ 07103, USA

\section{Springer}

(c) 2014 Del Re; licensee Springer. This is an Open Access article distributed under the terms of the Creative Commons Attribution License (http://creativecommons.org/licenses/by/4.0), which permits unrestricted use, distribution, and reproduction in any medium, provided the original work is properly credited.

\section{Review}

\section{Hippo signaling in Drosophila}

Determining how multicellular organisms attain and maintain appropriate organ size has plagued researchers and remains a major quest. Studies employing genetic mosaic screens in Drosophila melanogaster shed new light on this question and identified the core components of what is now known as the Hippo signaling pathway, appropriately named after Hippo kinase. Mutant flies genetically deficient for a functional Hippo (Hpo) [1-5], Salvador (Sav) [6,7] or Warts (Wts) [8,9] exhibited similar phenotypes consisting of robust tissue overgrowth, enhanced cell proliferation and suppressed apoptosis. These shared phenotypes suggested the involvement of each in a common signaling cascade that regulated organ size, which was subsequently demonstrated through additional genetic and protein interaction studies. Integral work revealed that Hippo (a serine/threonine kinase) interacts with Salvador (a WW-repeat protein and scaffold) and promotes the phosphorylation of both Salvador and Warts kinase [1]. A fourth core component, Mats (Mob as tumor suppressor) is also phosphorylated by Hippo and was found to play an important role in this cascade by interacting with Warts, which may further facilitate Warts activation $[10,11]$. These seminal studies demonstrated how the association and phosphorylation between core components promoted Hippo pathway activation. 
In 2005, work from Pan and colleagues provided the critical link between the Hippo cascade and gene expression. Using a yeast two-hybrid approach with Warts as bait, they were able to identify the transcription coactivator Yorkie (Yki) as a downstream target of Hippo signaling [12]. Subsequent work demonstrated that Yorkie is a direct substrate of Warts and that Yorkie promotes cell proliferation and growth. Importantly, inactivation of Yorkie was sufficient to prevent the overgrowth phenotype observed in Hippo pathway mutants, indicating that Yorkie is the major downstream effector of the pathway $[12,13]$. Yorkie itself lacks the ability to bind DNA directly and therefore influences gene expression through its interaction with the transcription factor Scalloped (Sd) [14-17]. Scalloped was demonstrated to mediate Yorkie-triggered overgrowth; however, overexpression of Scalloped alone did not elicit overgrowth, suggesting that Yorkie regulation is the critical factor for determining gene expression downstream of Hippo signaling [14].

\section{Hippo signaling in mammals}

There is high conservation of Hippo signaling components between Drosophila and mammals. Similar to the fly, mammalian Hippo signaling involves the activation of a conserved kinase cascade that ultimately phosphorylates the transcription co-activator Yap (Yes-associated protein) and its paralog TAZ (transcriptional co-activator with a PDZ-binding motif; both are orthologs of Drosophila Yki), thereby regulating downstream gene expression, cell proliferation and organ growth (for excellent reviews, see [18-22]). The core components of the mammalian Hippo pathway include the serine/threonine kinases Mst1/2 (mammalian sterile 20-like kinase; ortholog of Drosophila Hpo) and Lats1/2 (large tumor suppressor; ortholog of Drosophila Wts), as well as the adapter proteins Salvador (Sav1; also referred to as WW45) and Mob1 (Mps one binder; ortholog of Drosophila Mats)[23]. Analogous to Hippo signaling in the fly, Mst1/2 associates with and phosphorylates Sav1, which further promotes Mst1/2 activation. Once activated, Mst1/2 directly phosphorylates and activates Lats1/2[24]. Mst1/2 can also phosphorylate Mob1, which may further promote pathway activation through a currently undefined mechanism. Interestingly, Hippo signaling appears to have a high degree of evolutionary conservation from flies to humans as mammalian orthologs exist for each fly counterpart and generally function in a similar manner. However, it should be noted that recent work has demonstrated a clear divergence between select upstream pathway components (e.g., Drosophila Fat and Expanded, see below) and may explain why regulation of core Hippo signaling by these orthologs appears inconsistent between flies and mammals [25]. Furthermore, Yorkie/Yap transcriptional differences have been observed between flies and mammalian cell types highlighting differences in Hippo signaling outputs between species [26].

\section{Regulation of gene expression by the Hippo pathway}

Like many other established signal transduction pathways, Hippo signaling regulates a transcriptional program. The identification of Yorkie as a direct substrate of Warts provided the important link between the Hippo core components and gene expression [12,13,27]. As mentioned above, Yorkie does not bind DNA and partners with Scalloped to mediate gene expression, cell proliferation and survival [15-17]. Several gene targets of Hippo signaling have been identified in Drosophila, including cyclin E, diap1, and the microRNA bantam, which may modulate cell proliferation and survival $[1,12,14,28,29]$. Similarly, the Yorkie orthologs Yap/TAZ cannot bind DNA directly and modulate gene expression through transcription factor interaction. Perhaps the most established are the TEAD1-4 (TEA domain family member) transcription factors (orthologs of Drosophila $\mathrm{Sd}$ ), which have been shown to mediate many of the cellular effects of activated Yap/TAZ [17,30-44]. In addition to transcriptional partnering with TEADs, Yap/TAZ have the ability to co-activate or co-repress other known transcription factors including p73 [45], ErbB-4 [46], Runx [47,48], FoxO1 [49], Tbx5 [50] and Smads [51-53]; however their respective roles in Hippo signaling are not clear.

Yap has been the focus of intense research since it was discovered to be the link between Hippo signaling and gene expression. Yap contains five potential Lats phosphorylation sites (HXRXXS) and mutation of one of these sites, a serine to alanine substitution at residue 127, was shown to attenuate Yap phosphorylation by Lats2 and modulate its subcellular distribution from cytosolic to nuclear prevalence $[13,27,54,55]$. Serine 127 phosphorylation promotes 14-3-3 binding and Yap cytosolic retention and led to a decrease in Yap-TEAD interaction, although this modification did not directly alter Yap-TEAD binding [13]. Additional phosphorylation of Yap at serine 381 serves as a phosphodegron and promotes its proteosomal degradation [56]. Interestingly, recent work has shown that PKA-mediated phosphorylation of Lats2 promotes its kinase activity toward Yap, but is selective for serine 381, thereby enhancing Yap degradation [57]. These findings suggest that distinct signaling may bias Lats-mediated phosphorylation and subsequent regulation of Yap.

Yap activity is also regulated by multiple upstream signaling pathways that are independent of Hippo signaling. Yap was originally discovered as a WW-domain-containing protein that interacted with Yes, a member of the Src family of tyrosine kinases [58]. Subsequent work has shown that Yap is phosphorylated by Yes/Src and that this is required to enable Yap modulation of Runx2-mediated transcription [48]. Yap/TAZ also display extensive crosstalk 
with Wnt/ $\beta$-catenin signaling. Activation of Hippo signaling and increased phosphorylation of Yap/TAZ has been shown to promote their interaction with $\beta$-catenin, increasing cytosolic retention of $\beta$-catenin, and thereby negatively regulating Wnt/ $\beta$-catenin signaling [59]. Additionally, inhibition of Hippo and activation of Yap caused increased $\beta$-cateninmediated gene expression in the heart, and $\beta$-catenin was found to be required for the activated Yap phenotype [60]. Similar findings were observed using a constitutively active Yap mutant - namely that active Yap promotes $\beta$-catenin target gene expression both directly and indirectly (through activation of IGF-1 signaling) and that $\beta$-catenin is a critical mediator of Yap function [61]. These findings suggest that Yap is a positive regulator of the $\mathrm{Wnt} / \beta$-catenin pathway. Conversely, studies have also demonstrated that Wnt/ $\beta$ catenin signaling regulates the function of Yap/TAZ. Activation of Wnt causes stabilization of Yap through its target tribbles homolog 2 (TRIB2) [62]. Wnt can also prevent degradation of TAZ by preventing the phosphorylation of $\beta$-catenin, which serves as a bridge that links TAZ to its ubiquitin ligase $\beta$-TrCP [63]. Recently published work has further clarified the underlying mechanism by demonstrating that Yap/TAZ are integral components of the $\beta$-catenin destruction complex and that Wnt activation triggers the nuclear aggregation of both $\beta$-catenin and Yap/TAZ [64]. Additional reports have implicated the involvement of JNK in regulating Yap activity either directly through JNK phosphorylation of Yap [65] or indirectly by promoting the interaction of Lats with Ajuba family proteins thereby limiting the Lats-Yap inhibitory interaction [66]. Taken altogether, these studies highlight the crosstalk surrounding Yap/TAZ and suggest they are key nodes of signaling transduction.

\section{Upstream modulators of Hippo signaling in Drosophila}

As the importance of Hippo signaling becomes increasingly apparent, significant efforts have been made to clarify the proximal signaling responsible for pathway modulation. In Drosophila, a number of potential upstream components have been identified as important pathway regulators. Genetic screens identified the FERM domain family members Merlin (Mer) and Expanded (Ex). Mutations in either of these genes led to an attenuation of Hippo signaling in flies [67]. Interestingly, Expanded was shown to negatively regulate Yorkie directly through a proteinprotein interaction, which led to cytosolic sequestration and increased Yorkie degradation [68,69]. The mechanism responsible for linking Merlin to the core Hippo kinase cassette remained elusive until elegant work from Pan and colleagues revealed a potential mechanism. Their study found that membrane-associated Merlin binds and recruits Warts to the plasma membrane. Here, Warts is phosphorylated by an independent Hippo/Salvador complex, thereby promoting pathway activation. Of note, this work was unique in demonstrating spatial compartmentalization of this signaling pathway [24]. Additional studies have implicated Kibra, a protein known to interact with both Merlin and Expanded, as a potential regulator of Hippo signaling. Indeed, loss of Kibra function appears to phenocopy mer and ex mutants, while Kibra overexpression caused increased phosphorylation of Hippo, Warts and Yorkie [70-72]. Fat, an atypical protocadherin, has also been identified as an upstream regulator, and possible membrane receptor, of Hippo signaling in the fly [73-77]. Fat mutants (loss-of-function) displayed overgrowth phenotypes similar to those of Hippo pathway loss-of-function mutants, as well as dysregulation of Hippo phosphorylation and Yorkie target gene expression [73-75]. Furthermore, Fat modulated protein levels and proper localization of Expanded, identifying a potential mechanism that links Fat to Hippo. Daschous, another transmembrane cadherin, also regulates Hippo signaling through its interaction with Fat and Zyxin, ultimately modulating Warts protein levels [78]. Crumbs, a known cell polarity factor in Drosophila, has also been implicated in Hippo signaling $[53,79,80]$. Loss of Crumbs caused mislocalization of Expanded and inhibition of Hippo activation, leading to hyperactivation of Yorkie and increased tissue growth $[81,82]$. It has also been demonstrated that Hippo is phosphorylated in its activation loop by Tao-1, another member of the sterile 20like kinase family. Phosphorylation of Hippo by Tao-1 promoted the activation of core Hippo components and led to restrained Yorkie function [83,84]. Recent work from Wehr et al. employed a genome-wide RNAi screen in Drosophila and identified the salt-inducible kinases, Sik2 and Sik3, as negative regulators of Hippo signaling [85]. The Siks were found to disrupt Hippo/Warts complex formation through phosphorylation of Salvador, leading to increased Yap activation. As these kinases may be sensitive to nutrient sensing, they could provide a link between extracellular cues and growth through the Hippo pathway. Yet another kinase, homeodomain-interacting protein kinase 2 (HIPK2) has been demonstrated to regulate Hippo pathway activity. However, HIPK2 does not appear to modulate upstream Hippo members, but instead directly phosphorylates and activates Yorkie to promote increased tissue growth [86,87]. Given the intense interest in Hippo signaling, this burgeoning list of pathway components continues to expand. In this regard, for a more in-depth discussion of additional regulators of the Hippo pathway, the reader is referred to these excellent reviews [21,88-92].

\section{Upstream modulators of mammalian Hippo signaling}

Although many Drosophila Hippo pathway components have mammalian orthologs, and although many of these 
have been demonstrated to have similar functions to their fly counterparts, great care must be exercised when investigating Hippo signaling in mammalian systems as it most likely will prove to be more complex and nuanced. In fact, clear differences between flies and mammals with regard to Hippo signaling have been demonstrated $[25,26,93]$. Nonetheless, substantial effort has been directed toward elucidating the proximal regulators of mammalian Hippo signaling in recent years and significant progress has been made on this front. RASSF1A (Ras association domain family 1A) is a tumor suppressor and scaffold protein that has been shown to interact with Mst1/2 and Sav1 to promote activation of Hippo signaling [94,95]. RASSF1A causes increased Mst1/2 autophosphorylation, at least in part through suppression of Mst1/2 dephosphorylation by PP2A [96], thereby promoting Mst1/2 activation and subsequent apoptosis $[97,98]$. It should be noted that the Drosophila ortholog of RASSF1A (dRASSF) was demonstrated to prevent Hippo activation [93], a reminder of the potential differences between flies and mammals. Studies involving the tumor suppressor NF2 (Neurofibromin 2; ortholog of Merlin) have demonstrated its role in regulating mammalian Hippo signaling. Targeted deletion of the Nf2 gene in the mouse liver (albumin-Cre driver specific for hepatocytes and biliary cells) led to increased Yap activation, increased cell proliferation, increased liver size and eventual hepatocellular carcinoma (HCC) $[99,100]$. Interestingly, this NF2 KO phenotype was completely prevented in mice that were also haplo-deficient for Yap, indicating that augmented Yap activity is responsible for this pathology [99]. On the other hand, treatment with an EGFR (epidermal growth factor receptor) inhibitor also prevented the overgrowth caused by Nf2 deletion, suggesting that multiple pathways downstream of NF2 may be altered and contribute to HCC [100]. Loss of NF2 function in the dorsal telencephalon of the murine brain also elicited increased Yap/TAZ activation and increased cell proliferation/expansion leading to developmental defects [101]. While these results implicate a role for NF2 in regulating Hippo signaling in two distinct tissues, additional work has demonstrated a similar anti-proliferative but non-Hippo-related effect. Specifically, NF2 was found to translocate to the nucleus where it inhibited the E3 ligase DCAF1 to suppress cell proliferation independent of Hippo signaling [102]. Taken together, RASSF1A and NF2 appear to be positive modulators that promote mammalian Hippo signaling in multiple tissue types.

The ability to sense and transduce extracellular signals and/or cues from the surrounding environment into the cell is a hallmark of traditional signaling pathways. Along these lines, work from Guan and colleagues demonstrated that cell density regulates the activation status of Hippo signaling [13]. Cell-to-cell contact, long known to cause inhibition of proliferation, was linked to the inactivation of Yap. Importantly, this work demonstrated the involvement of the Hippo pathway in regulating cell proliferation in response to the cell density signal. Further studies revealed that $\alpha$-catenin is an important mediator of the cell density signal and a direct regulator of Yap phosphorylation and degradation in epithelial stem cells [39]. In a similar vein, Kim et al. reported that E-cadherin regulates Yap exclusion from the nucleus in a cell-density-dependent context [103]. Yap inhibition and cytosolic retention caused by cell-cell contact was mediated by E-cadherin, and forced Yap expression was able to overcome the anti-proliferative effect of E-cadherin. For excellent reviews regarding Hippo regulation by cell-cell contact and cell polarity see $[91,104]$.

In a landmark study, the Guan group was the first to demonstrate that extracellular receptors, in this case GPCRs (G-protein-coupled receptors), could modulate Hippo signaling [105]. Ligands that preferentially couple

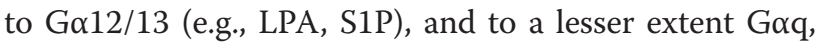
were shown to inhibit Hippo signaling and promote Yap activation. Conversely, ligands that preferentially activate Gas (e.g., epinephrine) stimulated Hippo signaling and inhibited Yap activity. Follow-up work described similar findings for PARs (protease-activated receptors), a subset of GPCRs that is stimulated by the endogenous ligand thrombin [106]. Further independent work confirmed that the extracellular diffusible signals LPA and S1P are indeed potent activators of Yap [107]. Interestingly, these ligands appear to act through the actin cytoskeleton and the small G-protein RhoA, as the signaling cascade is sensitive to actin disruption and inhibition of RhoA, and regulate the function of Lats kinases to control Yap activity $[105,107,108]$. Further investigation into the connection between cytoskeletal integrity and Lats identified a role for PKA (cAMP-dependent protein kinase) in the regulation of Hippo signaling. cAMP/PKA were shown to cause Yap/TAZ phosphorylation and inhibition through a process requiring actin polymerization and RhoA [57,108]. Kim et al. also demonstrated direct phosphorylation and activation of Lats2 by PKA. This modification appeared to bias Lats2 kinase activity toward inhibitory phosphorylation of Yap at serine 381 but not serine 127 [57]. In addition to GPCR-dependent modulation of Hippo signaling, two compelling studies demonstrated a role for receptor tyrosine kinases (RTKs) in regulating this cascade. Fan et al. demonstrated that activation of the EGF receptor in mammary cells caused the activation of Yap [109]. Mechanistically, the authors demonstrated that this was mediated by PI3K and PDK1, which are activated downstream of EGF receptor engagement, but did not require AKT. Instead, PDK1 associates with Salvador to disrupt the core Hippo signaling components, leading to Lats inhibition and Yap activation. A similar finding was reported by Reddy and 


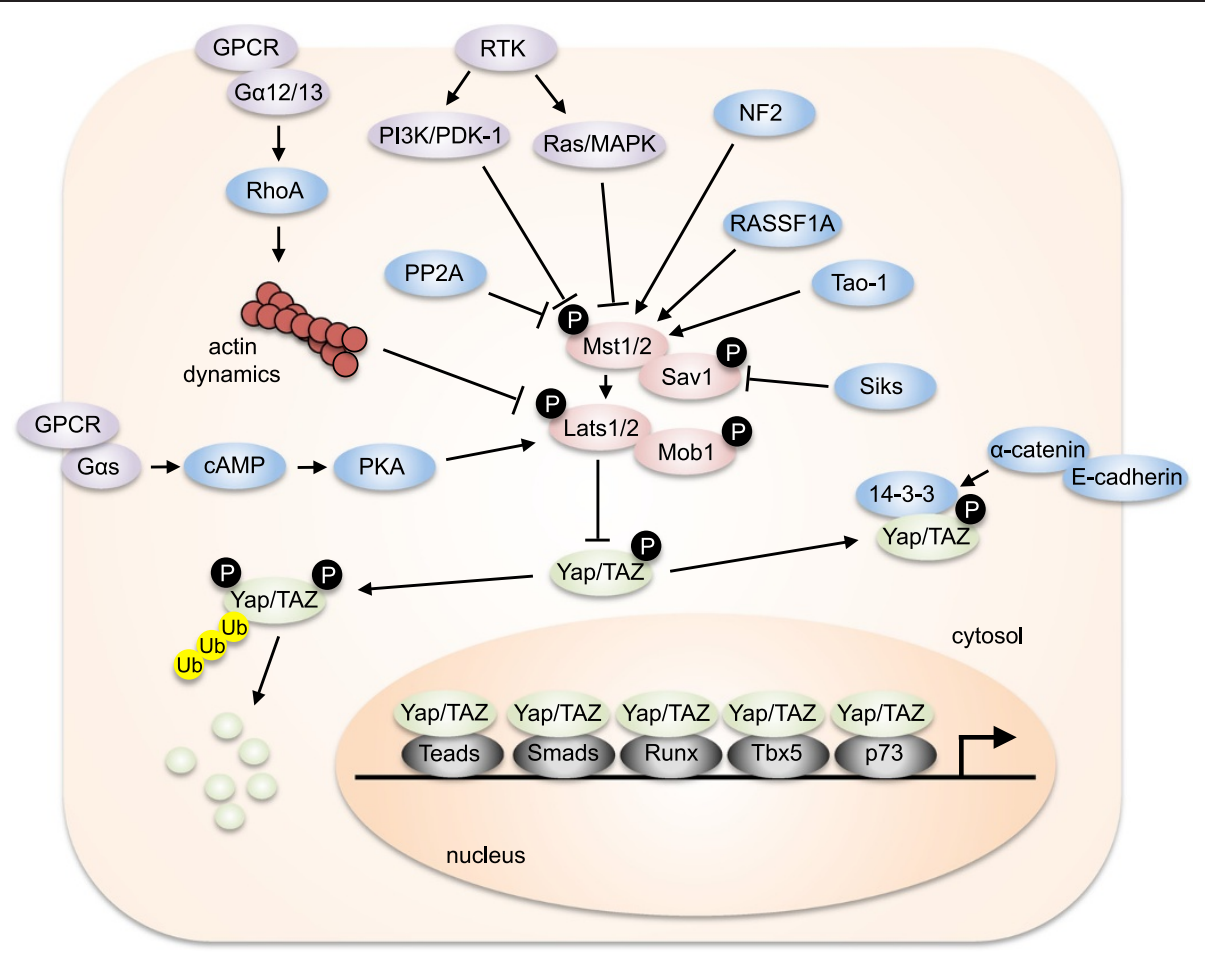

Figure 1 A schematic representation of mammalian Hippo signaling. The core components of the Hippo pathway in mammals consist of Mst1/2, Lats1/2, Salvador (Sav1) and Mob1. Active Lats1/2 phosphorylates and inhibits Yap/TAZ. Yap phosphorylation on Serine 127 results in 14-3-3 binding and cytosolic retention (right side) while Serine 381 phosphorylation leads to proteosomal degradation (left side). Nuclear Yap/ TAZ regulate gene expression resulting in proliferation, growth and survival. Yap/TAZ association with several identified transcription factors is shown. Arrows and blunt lines indicate activation and inhibition, respectively.

Irvine [110]. Here, activation of EGFR elicited Ras/ MAPK activation and phosphorylation of the Ajuba family protein WTIP (Wilms tumor protein 1-interacting protein). The fly homolog of WTIP, Jub, was also phosphorylated by MAPK, causing it to bind to Salvador and Warts, inhibiting Warts and eliciting Yorkie activation (see Figure 1).

Additional groundbreaking studies from the Piccolo lab have shown that extracellular matrix (ECM) stiffness and mechanotransduction systems can also modulate the activity of Yap/TAZ [111]. This work demonstrated that Yap/TAZ are a critical link between ECM rigidity and altered gene expression, which is known to affect cell growth, differentiation and proliferation. The cell's ability to spread and the amount of mechanical stress to which it is subjected were identified as key regulators of Yap/TAZ nuclear localization and transcriptional activity. The underlying mechanism was found to be mediated by RhoA and actomyosin tension; however, Mst/ Lats kinases appeared to be dispensable. Further work using a siRNA screen of F-actin-associated proteins identified and subsequently confirmed Cofilin, CapZ and Gelsolin as important inhibitors of Yap/TAZ in cells experiencing low mechanical stress [112]. Taken altogether, regulation of mammalian Hippo signaling is complex and likely incorporates multiple upstream inputs to determine intensity and duration of signal activation. Furthermore, it is likely that regulation and function of this pathway are cell- and tissue-type-specific and additional investigation is therefore needed.

\section{Hippo signaling and heart development}

The mammalian heart develops from mesodermal tissues, first forming a heart tube, which then loops and gives rise to atrial and ventricular components and an outflow tract. These complex processes require finely orchestrated spatial-temporal signaling events between multiple cell types to give rise to a normal, healthy heart [113]. Tbx 5 is a transcription factor and one of several known regulators of embryonic heart development [114]. Importantly, Tbx5 can be co-activated by Yap/ TAZ, indicating the possible involvement of Hippo signaling in early stage heart development [50].

Recent studies have begun to further examine the role of Hippo in mammalian cardiogenesis. A mouse model harboring embryonic deletion of Sav1, Mst1/2 or Lats2 in the heart revealed the importance of these Hippo components for proper organ development [60]. All knockout lines displayed a similar phenotype and showed hyperproliferation of cardiomyocytes with 
thickened ventricular walls due to an excessive cardiomyocyte number. These mice also died prior to birth. Mechanistically, there was significant upregulation of Wnt target gene expression, which was rescued by crossing the Hippo mutant mice with those deficient for $\beta$ catenin. These findings suggest that Hippo restrains cardiomyocyte proliferation and heart size by inhibiting Wnt signaling and point to the importance of Hippo signaling during cardiogenesis. In a complimentary approach, two additional groups utilized genetically altered mice that harbored embryonic deletion of Yap in the heart to interrogate the function of endogenous Yap during heart development $[61,115]$. Importantly, two different promoters were used to drive cardiomyocyte-specific Cre recombinase expression; however, similar results were observed between the two lines including a decrease in cardiomyocyte proliferation, a decrease in progrowth and proliferative gene expression (the former via Wnt/ $\beta$-catenin and the latter via TEAD), and embryonic lethality between E10.5-12.5. Taken together, these findings demonstrate the critical involvement of Hippo signaling during cardiogenesis and suggest that a balance of Yap activity is required for proper heart formation (see Table 1 for overview of Hippo-related mouse models and cardiac phenotypes).

\section{Hippo signaling in cardiac regeneration}

Embryonic heart growth occurs mainly through proliferation of cardiomyocytes, whereas shortly after birth these cells experience a dramatic reduction in their ability to divide. Thus, further organ growth is almost exclusively mediated through the enlargement of existing cardiomyocytes. Around this same time, the ability of the mammalian heart to regenerate following injury drastically declines. It should be noted, however, that low-level cardiomyocyte turnover seems to occur throughout adult mammalian life [116,117] and approaches aimed at exploiting this phenomenon are an active area of cardiovascular research. This is in contrast to the zebrafish, which is able to regenerate up to $20 \%$ of its cardiac mass following myocardial resection, even during adulthood [118]. Because the loss of cardiomyocytes in response to injury is a major contributor to impaired function and heart failure, it is fundamental to understand the mechanisms that regulate proliferation and consequently regeneration of adult mammalian cardiomyocytes. Yap has been demonstrated to promote the self-renewal and "stemness" of embryonic stem cells [119], satellite muscle cells [120] and liver cells [121], while suppressing or even reversing their differentiation, indicating that Yap could potentially be targeted to manipulate precursor cell fate and perhaps modify regenerative capacity.

Several studies have demonstrated the ability of Yap to promote proliferation of neonatal rat cardiomyocytes. By using adenoviral overexpression systems, these gain-offunction approaches upregulated active Yap and elicited increases in Ki-67-positive, phosphorylated histone H3positive, and BrdU-positive cardiomyocytes in culture

Table 1 Overview of Hippo-related mouse models

\begin{tabular}{|c|c|c|c|}
\hline Mouse line & Cardiac phenotype & Yap activity & References \\
\hline Mst1 Tg (aMHC) & $\begin{array}{l}\text { Dilated cardiomyopathy, increased apoptosis and no compensatory hypertrophy. } \\
\text { Premature death. }\end{array}$ & ND & {$[129]$} \\
\hline DN-Mst1 Tg (aMHC) & Normal cardiac function. Protection against I/R and MI. & ND & {$[129,130]$} \\
\hline Lats2 Tg (aMHC) & Baseline cardiac dysfunction. Reduced heart size. & ND & {$[135]$} \\
\hline DN-Lats2 Tg (aMHC) & Cardiac hypertrophy with normal cardiac function. Protection against I/R. & ND & {$[135]$} \\
\hline Sav1 cKO (Nkx2.5-cre) & Embryonic lethal. Cardiomegaly, thickened walls and increased proliferation. & Increased & {$[60]$} \\
\hline Mst1/2 cKO (Nkx2.5-cre) & Embryonic lethal. Enlarged heart. & ND & {$[60]$} \\
\hline Lats1/2 cKO (Nkx2.5-cre) & Embryonic lethal. Enlarged heart. & ND & {$[60]$} \\
\hline Yap Tg (aMHC) & Enlarged heart with increased proliferation. Increased cardiac regeneration. & Increased & {$[123]$} \\
\hline Yap Tg $(\beta M H C)$ & Enlarged heart with Increased proliferation. & Increased & {$[115]$} \\
\hline $\begin{array}{l}\text { Yap Tg (aMHC; ROSA26 } 6^{\mathrm{fs}-\mathrm{rtTA}} \text {; } \\
\text { TRE-Yap) }\end{array}$ & $\begin{array}{l}\text { Normal cardiac function. Increased proliferation. Protection against MI with } \\
\text { no change in apoptosis. }\end{array}$ & Increased & {$[124]$} \\
\hline Yap cKO (Nkx2.5-cre) & Embryonic lethal. Reduced heart size, reduced proliferation. & Reduced & {$[61,115]$} \\
\hline Yap cKO (Tnnt2-cre) & Embryonic lethal. Reduced heart size, reduced proliferation. & Reduced & {$[115]$} \\
\hline Yap cKO (aMHC-cre) & $\begin{array}{l}\text { Dilated cardiomyopathy, increased apoptosis, increased fibrosis and } \\
\text { premature death. Worsened outcome after MI (het). }\end{array}$ & Reduced & {$[122,123]$} \\
\hline Sav1 cKO (aMHC-MCM) & $\begin{array}{l}\text { Normal cardiac function. Increased proliferation and heart regeneration. } \\
\text { Protected against Ml. }\end{array}$ & Increased & {$[125]$} \\
\hline Lats1/2 cKO (aMHC-MCM) & $\begin{array}{l}\text { Normal cardiac function. Increased proliferation and heart regeneration. } \\
\text { Protected against MI. }\end{array}$ & Increased & {$[125]$} \\
\hline
\end{tabular}


[61,115,122]. Ectopic expression of activated Yap in vivo also caused increased cardiomyocyte proliferation, indicating that this observation is not limited or specific to neonatal cells $[115,123,124]$. Similarly, conditional deletion of Sav1 in the adult mouse heart, which causes increased Yap activation, also triggered increased cardiomyocyte proliferation [125], providing further evidence that Hippo signaling restrains adult cardiomyocyte renewal. Recent work has demonstrated that neonatal mouse hearts have a regenerative capacity similar to zebrafish during the first 7 days after birth (P1-7); however, following P7 this capacity for heart renewal declines dramatically [126]. To determine whether Hippo signaling regulates neonatal heart regeneration, Yap transgenic mice were generated. Increased cardiac Yap expression was able to protect against cardiomyocyte loss, fibrosis and scarring after chronic myocardial infarction (MI) in mice beginning at P7. Conversely, cardiac-specific deletion of Yap ablated the neonatal regenerative capacity observed in wild-type mice following MI. Yap CKO mice had larger scar areas while wild-type neonates appeared normal [123], suggesting that Yap is a critical mediator of heart regeneration shortly after birth. Additional studies utilized inducible cardiac Sav1 and Lats1/2 CKO mice to test whether these Hippo pathway components could also influence the regenerative capacity of the mammalian heart [125]. Conditional deletion of Sav1 or Lats1/2 in neonatal mice was shown to promote heart repair and extend the regenerative potential following cardiac resection at P8. Furthermore, Hippo deficiency led to increased cardiomyocyte proliferation and myocardial protection in response to MI either at P8 or in 8week-old adult mice. These Sav1 CKO mice also had improved cardiac function and smaller scars after MI compared to wild-type controls. In sum, these studies indicate that Hippo signaling is an important modulator of cardiomyocyte proliferation and myocardial regeneration in response to injury.

\section{Hippo signaling in heart disease}

Mst1 was first cloned and characterized nearly 20 years ago and was later identified as a Hippo ortholog and a core component of the mammalian Hippo signaling pathway [127]. Mst1 is activated during apoptosis of cancer cell lines [128] and cardiomyocytes [129] and, importantly, contributes to their programmed cell death. Myocardial Mst1 transgene expression driven by the cardiomyocyte-specific $\alpha \mathrm{MHC}$ promoter led to a profound cardiac phenotype in mice [129]. Mst1 transgenic (Tg) mice developed severe dilated cardiomyopathy at a young age and died prematurely due to heart failure. These $\mathrm{Tg}$ mice had elevated caspase activation and cardiomyocyte apoptosis, which contributed to ventricular wall thinning and a decline in cardiac function. On the other hand, using the same approach to express a kinase-inactive (K59R) DN-Mst1 in the heart inhibited endogenous Mst1 activity and afforded cardioprotection [129]. During ischemia and reperfusion (I/R), which simulates acute coronary occlusion and subsequent restoration of blood flow, Mst1 is activated and contributes to cardiomyocyte apoptosis. Indeed, DN-Mst1 $\mathrm{Tg}$ mice showed significantly fewer TUNEL-positive cardiomyocytes and significantly reduced scar formation following $\mathrm{I} / \mathrm{R}$ [129]. Wild-type non-transgenic (NTg) and DN-Mst1 $\mathrm{Tg}$ mice were also subjected to chronic MI. DN-Mst1 Tg mice had less apoptosis, reduced fibrosis, attenuation of left ventricle dilation and improved cardiac function compared to controls [130]. Autophagy, an established adaptive mechanism that can protect the heart during stress [131], is also regulated by Mst1 in the adult mouse heart. Recent work demonstrated that Mst1-mediated phosphorylation of Beclin-1 promotes the association of Beclin-1 with Bcl-2 and thereby inhibits cardiomyocyte autophagy [132]. This inhibitory mechanism translates to increased myocardial injury in response to prolonged ischemia. Another recently identified target of Mst1 is the pro-survival Bcl-2 family member Bcl-xL. During I/R, Mst1 was found to translocate to mitochondria and phosphorylate $\mathrm{Bcl}-\mathrm{xL}$, causing its dissociation from Bax and leading to Bax activation and cardiomyocyte apoptosis [133]. These findings provide novel mechanisms to explain how Mst1 elicits detrimental outcomes in the heart. Work in pancreatic beta cells has also demonstrated a deleterious role of Mst1 through the promotion of beta cell apoptosis and dysfunction that could contribute to insulin resistance [134]. Together these findings suggest that Mst1 could be a valid therapeutic target not only for the treatment of heart disease, but diabetes as well.

Similar to Mst1, increased expression of Lats2 promotes, while expression of DN-Lats2 attenuates, apoptosis of cultured cardiomyocytes [135]. Interestingly, Lats2 Tg mice had increased myocardial fibrosis and depressed cardiac function, while no significant difference in cardiomyocyte apoptosis was observed under basal conditions. Transgenic expression of DN-Lats2 was used to inhibit endogenous Lats 2 in the mouse heart. In response to pressure overload stress (an experimental model of hypertension), DN-Lats2 $\mathrm{Tg}$ mice were protected against cardiomyocyte apoptosis and showed an enhanced hypertrophic response, i.e., individual cardiomyocyte enlargement leading to greater overall heart mass [135]. DN-Lats2 $\mathrm{Tg}$ mice were also protected against $I / R$ injury and had significantly lower levels of cardiomyocyte apoptosis and scar formation after $I / R$, which was mediated through increased Yap activation [49]. Taken together, these findings implicate Mst1 and Lats2 as critical mediators of myocardial injury and dysfunction following both acute and prolonged stress. 
RASSF1A associates with Mst1/2 and promotes mammalian Hippo signaling [94-98]. In the heart, two separate groups utilizing independently generated RASSF1A KO mouse lines reported very similar observations $[136,137]$. RASSF1A KO mice had no overt cardiac phenotype at baseline; however, in response to pressure overload, cardiomyocyte hypertrophy was augmented compared to wild-type mice. No apparent differences in cardiac function were observed between wild-type and RASSF1A KO mice. Interestingly, cardiac fibrosis was enhanced in RASSF1A KO mice following pressure overload [136,137]. Further comparison between systemic RASSF1A KO mice and cardiac-specific RASSF1A CKO mice revealed a paracrine mechanism involving TNF- $\alpha$ secretion by cardiac fibroblasts that mediated the enhanced growth and fibrosis observed in RASSF1A KO hearts [137]. Importantly, RASSF1A CKO mice had better cardiac function after pressure overload compared to controls, indicating that RASSF1A function is most likely cell-type-dependent. RASSF1A was also shown to promote Mst1 activation both in cultured cardiomyocytes and the mouse heart, which mediated its pro-apoptotic effect [137]. Therefore, it appears that RASSF1A is an important upstream regulator of Mst1 in the mammalian heart, but the overall impact of RASSF1A on the myocardium is a balance between cell-type-specific signaling and interactions.

Yap is an oncogene that can promote tumorigenesis $[39,138-144]$, yet its function in the adult heart has only recently been investigated. Systemic deletion of Yap results in embryonic lethality at day E8.5 $[145,146]$. Similarly, disruption of Tead1 also causes embryonic death around E11-12 [138]. Interestingly, Tead1-/- embryos showed severe cardiac abnormalities including enlarged pericardial cavities, thin ventricular walls and reduced number of trabeculae [145]. These findings suggest that Yap and TEAD1 may have redundant functions during early embryonic development, one of which may be the regulation of heart formation in mice. To avoid embryonic lethality and allow for the study of Yap function in later stages of development, tissue-specific Yap deletion has been attained and employed [99]. Myocardial postnatal deletion of Yap caused a rapid and severe cardiomyopathy with premature death by $10-12$ weeks of age $[122,123]$. Yap CKO mice had robust increases in cardiomyocyte apoptosis and cardiac fibrosis that were associated with decreased cardiac function, indicating a critical role for Yap in maintaining adult heart homeostasis [122]. TAZ CKO mice have also been generated [123]. Interestingly, postnatal cardiac TAZ deletion did not result in an obvious phenotype and did not appear to alter lifespan. Similarly, hemizygous myocardial Yap deletion $(\mathrm{Yap}+/-)$ did not elicit a baseline cardiac phenotype compared to control mice [122]. However, following chronic MI stress, during which Yap is activated at the site of injury, Yap+/- mice showed increased cardiomyocyte apoptosis and fibrosis. Furthermore, Yap+/- hearts had significantly fewer proliferating cardiomyocytes, attenuated cardiomyocyte compensatory hypertrophy, and worsened heart function compared to control mice after MI [122]. Conversely, transgenic expression of activated Yap in mouse hearts, either prior to or immediately after MI, protected against MI-induced injury [123,124]. Increased Yap expression in adult mouse hearts caused an increase in cardiomyocyte proliferation and significantly reduced scar formation $[123,124]$. These findings are comparable to those observed in Sav1 CKO hearts after MI, a condition that upregulates Yap activity [125]. Taken altogether, these results indicate that Yap is required for adult heart homeostasis and is strongly cardioprotective, likely through its ability to promote both cardiomyocyte survival and proliferation in response to injury.

\section{Exploiting Hippo signaling for heart regeneration}

Because Yap activation has been shown to 1) regulate fetal heart growth $[60,61,115], 2)$ promote cardiomyocyte proliferation in both neonatal and adult cardiomyocytes in vivo [115,122-124] and 3) extend the regenerative capacity of the neonatal mouse heart $[123,125]$, Hippo signaling has become an intriguing potential target of manipulation for heart regeneration. However, sizable challenges exist. Stimulating adult cardiomyocytes to reenter the cell cycle and proliferate is a major barrier. Even a signal as potent as activated Yap expression cannot drive a subpopulation of adult cardiomyocytes toward division at a rate that is likely to have much regenerative impact. Indeed, Yap-mediated success in neonatal cardiomyocytes is much greater, indicating that fundamental differences emerge with age. Similarly, stimulating Yap activity through Hippo loss of function had comparable effects on adult cardiomyocyte proliferation, raising the question of whether this approach will be viable and how it might be enhanced. Another concern is the potential for tumorigenesis as a result of increased Yap activation since its oncogenic potential has been demonstrated in proliferative tissues. Although the use of stem cells and cardiac progenitor cells for the treatment of MI in patients has been controversial, this remains another potential route of exploration for Hippo manipulation. Yap has been shown to influence stem/ progenitor cell potency and differentiation, yet its function in adult cardiac progenitors is not known. It is possible that engineering cardiac progenitors through modulation of Hippo signaling could afford a more robust progenitor population and increased therapeutic benefit. Additionally, the level of Yap expression/activation in adult cardiac progenitor cells is not known and it would be interesting to determine whether Yap could serve as a marker of cardiac progenitors, similar to c-kit, 
Islet-1, Sca-1 etc., that proves even more efficacious and overcomes the limitations of those populations being isolated and used currently [147].

\section{Conclusions}

The Hippo signaling pathway has risen to prominence and is regarded as an important transduction mechanism that regulates cell growth, proliferation and survival. It has important implications for physiology and pathology that are the focus of intense ongoing research. Studies to elucidate the importance of Hippo signaling in cardiovascular biology and disease continue to shed light and reveal further complexity of this cascade in the mammalian heart. Future work may focus on the following points of outstanding interest: 1) the underlying mechanism explaining how Yap promotes cardiomyocyte proliferation; 2) upstream regulation of Hippo and how various stresses modulate the pathway; 3) possible crosstalk between additional established signaling networks that impact the heart; and 4) targeting Hippo components using small molecules to influence signaling and outcomes.

\begin{abstract}
Abbreviations
BrdU: Bromodeoxyuridine; CAMP: Cyclic adenosine monophosphate: ECM: Extracellular matrix; EGFR: Epidermal growth factor receptor; Ex: Expanded; GPCR: G-protein coupled receptor; HCC: Hepatocellular carcinoma; HIPK2: Homeodomain interacting protein kinase; Hpo: Hippo; I/R: Ischemia/reperfusion; Lats: Large tumor suppressor; LPA: Lysophosphatidic acid; MAPK: Mitogen-activated protein kinase; Mats: Mob as tumor suppressor; Mer: Merlin; Ml: Myocardial infarct; Mob: Mps one binder; Mst: Nammalian sterile 20-like kinase; NTg: Non-transgenic; PAR: Protease activated receptor; PKA: CAMP-dependent protein kinase; RASSF1A: Ras association domain family; RTK: Receptor tyrosine kinase; S1P: Sphingosine-1-phosphate; Sav: Salvador; Sd: Scalloped; TAZ: Transcriptional co-activator with a PDZ-binding motif; TEAD: TEA domain family member; Tg: Transgenic; TUNEL: Terminal deoxynucleotidyl transferase dUTP nick end labeling; WTIP: Wilms tumor protein 1-interacting protein; Wts: Warts; Yap: Yes-associated protein; Yki: Yorkie.
\end{abstract}

\section{Competing interest}

There are no commercial or other competing interests to disclose.

\section{Acknowledgements}

Thanks to Junichi Sadoshima for helpful discussion and Christopher D. Brady for critical reading of the manuscript. This work was funded by grants from the NIH (HL122669) and the American Heart Association (11SDG7240066).

Received: 28 April 2014 Accepted: 22 July 2014

Published online: 16 September 2014

\section{References}

1. Wu S, Huang J, Dong J, Pan D: hippo encodes a Ste-20 family protein kinase that restricts cell proliferation and promotes apoptosis in conjunction with salvador and warts. Cell 2003, 114:445-456.

2. Harvey KF, Pfleger CM, Hariharan IK: The Drosophila Mst ortholog, hippo, restricts growth and cell proliferation and promotes apoptosis. Cell 2003, 114:457-467.

3. Udan RS, Kango-Singh M, Nolo R, Tao C, Halder G: Hippo promotes proliferation arrest and apoptosis in the Salvador/Warts pathway. Nat Cell Biol 2003, 5:914-920

4. Pantalacci S, Tapon N, Leopold P: The Salvador partner Hippo promotes apoptosis and cell-cycle exit in Drosophila. Nat Cell Biol 2003, 5:921-927.

5. Jia J, Zhang W, Wang B, Trinko R, Jiang J: The Drosophila Ste20 family kinase dMST functions as a tumor suppressor by restricting cell proliferation and promoting apoptosis. Genes Dev 2003, 17:2514-2519.
6. Tapon N, Harvey KF, Bell DW, Wahrer DC, Schiripo TA, Haber DA: salvador Promotes both cell cycle exit and apoptosis in Drosophila and is mutated in human cancer cell lines. Cell 2002, 110:467-478.

7. Kango-Singh M, Nolo R, Tao C, Verstreken P, Hiesinger PR, Bellen HJ: Shar-pei mediates cell proliferation arrest during imaginal disc growth in Drosophila. Development 2002, 129:5719-5730.

8. Justice RW, Zilian O, Woods DF, Noll M, Bryant PJ: The Drosophila tumor suppressor gene warts encodes a homolog of human myotonic dystrophy kinase and is required for the control of cell shape and proliferation. Genes Dev 1995, 9:534-546.

9. Xu T, Wang W, Zhang S, Stewart RA, Yu W: Identifying tumor suppressors in genetic mosaics: the Drosophila lats gene encodes a putative protein kinase. Development 1995, 121:1053-1063.

10. Lai ZC, Wei X, Shimizu T, Ramos E, Rohrbaugh M, Nikolaidis N: Control of cell proliferation and apoptosis by mob as tumor suppressor, mats. Cell 2005, 120:675-685.

11. Wei X, Shimizu T, Lai ZC: Mob as tumor suppressor is activated by Hippo kinase for growth inhibition in Drosophila. Embo J 2007, 26:1772-1781.

12. Huang J, Wu S, Barrera J, Matthews K, Pan D: The Hippo Signaling Pathway Coordinately Regulates Cell Proliferation and Apoptosis by Inactivating Yorkie, the Drosophila Homolog of YAP. Cell 2005, 122:421-434.

13. Zhao B, Wei X, Li W, Udan RS, Yang Q, Kim J, Xie J, Ikenoue T, Yu J, Li L, Zheng P, Ye K, Chinnaiyan A, Halder G, Lai ZC, Guan K L: Inactivation of YAP oncoprotein by the Hippo pathway is involved in cell contact inhibition and tissue growth control. Genes Dev 2007, 21:2747-2761.

14. Wu S, Liu Y, Zheng Y, Dong J, Pan D: The TEAD/TEF family protein Scalloped mediates transcriptional output of the Hippo growthregulatory pathway. Dev Cell 2008, 14:388-398.

15. Zhang $L$, Ren F, Zhang $Q$, Chen $Y$, Wang B, Jiang J: The TEAD/TEF family of transcription factor Scalloped mediates Hippo signaling in organ size control. Dev Cell 2008, 14:377-387.

16. Goulev Y, Fauny JD, Gonzalez-Marti B, Flagiello D, Silber J, Zider A: SCALLOPED interacts with YORKIE, the nuclear effector of the hippo tumor-suppressor pathway in Drosophila. Curr Biol 2008, 18:435-441.

17. Zhao B, Ye X, Yu J, Li L, Li W, Li S, Lin JD, Wang CY, Chinnaiyan AM, Lai ZC, Guan KL: TEAD mediates YAP-dependent gene induction and growth control. Genes Dev 2008, 22:1962-1971.

18. Pan D: The hippo signaling pathway in development and cancer. Dev Cell 2010, 19:491-505.

19. Chan SW, Lim CJ, Chen L, Chong YF, Huang C, Song H: The Hippo pathway in biological control and cancer development. J Cell Physiol 2011, 226:928-939.

20. Yu FX, Guan KL: The Hippo pathway: regulators and regulations. Genes Dev 2013, 27:355-371.

21. Lin Jl, Poon CL, Harvey KF: The Hippo size control pathway-ever expanding. Sci Signal 2013, 6:pe4.

22. Avruch J, Zhou D, Fitamant J, Bardeesy N, Mou F, Barrufet LR: Protein kinases of the Hippo pathway: regulation and substrates. Semin Cell Dev Biol 2012, 23:770-784.

23. Pan D: Hippo signaling in organ size control. Genes Dev 2007, 21:886-897.

24. Yin F, Yu J, Zheng Y, Chen Q, Zhang N, Pan D: Spatial organization of Hippo signaling at the plasma membrane mediated by the tumor suppressor Merlin/NF2. Cell 2013, 154:1342-1355.

25. Bossuyt W, Chen CL, Chen Q, Sudol M, McNeill H, Pan D: An evolutionary shift in the regulation of the Hippo pathway between mice and flies. Oncogene 2014, 33:1218-1228.

26. Zhang X, Milton CC, Humbert PO, Harvey KF: Transcriptional output of the Salvador/warts/hippo pathway is controlled in distinct fashions in Drosophila melanogaster and mammalian cell lines. Cancer Res 2009, 69:6033-6041.

27. Dong J, Feldmann G, Huang J, Wu S, Zhang N, Comerford SA: Elucidation of a universal size-control mechanism in Drosophila and mammals. Cell 2007, 130:1120-1133.

28. Thompson BJ, Cohen SM: The Hippo pathway regulates the bantam microRNA to control cell proliferation and apoptosis in Drosophila. Cell 2006, 126:767-774

29. Nolo R, Morrison CM, Tao C, Zhang X, Halder G: The bantam microRNA is a target of the hippo tumor-suppressor pathway. Curr Biol 2006, 16:1895-1904.

30. Ota M, Sasaki H: Mammalian Tead proteins regulate cell proliferation and contact inhibition as transcriptional mediators of Hippo signaling. Development 2008, 135:4059-4069. 
31. Zhao B, Kim J, Ye X, Lai ZC, Guan KL: Both TEAD-binding and WW domains are required for the growth stimulation and oncogenic transformation activity of yes-associated protein. Cancer Res 2009, 69:1089-1098.

32. Pobbati AV, Chan SW, Lee I, Song H, Hong W: Structural and functional similarity between the Vgll1-TEAD and the YAP-TEAD complexes. Structure 2012, 20:1135-1140.

33. Bhat KP, Salazar KL, Balasubramaniyan V, Wani K, Heathcock L, Hollingsworth F: The transcriptional coactivator TAZ regulates mesenchymal differentiation in malignant glioma. Genes Dev 2011, 25:2594-2609.

34. Zhang H, Liu CY, Zha ZY, Zhao B, Yao J, Zhao S: TEAD transcription factors mediate the function of TAZ in cell growth and epithelial-mesenchymal transition. J Biol Chem 2009, 284:13355-13362.

35. Chan SW, Lim CJ, Loo LS, Chong YF, Huang C, Hong W: TEADs mediate nuclear retention of TAZ to promote oncogenic transformation. J Biol Chem 2009, 284:14347-14358.

36. Mahoney WM Jr, Hong JH, Yaffe MB, Farrance IK: The transcriptional co-activator TAZ interacts differentially with transcriptional enhancer factor-1 (TEF-1) family members. Biochem J 2005, 388:217-225.

37. Lamar JM, Stern P, Liu H, Schindler JW, Jiang ZG, Hynes RO: The Hippo pathway target, YAP, promotes metastasis through its TEAD-interaction domain. Proc Natl Acad Sci U S A 2012, 109:E2441-E2450.

38. Liu-Chittenden Y, Huang B, Shim JS, Chen Q, Lee SJ, Anders RA: Genetic and pharmacological disruption of the TEAD-YAP complex suppresses the oncogenic activity of YAP. Genes Dev 2012, 26:1300-1305.

39. Schlegelmilch K, Mohseni M, Kirak O, Pruszak J, Rodriguez JR, Zhou D: Yap1 acts downstream of alpha-catenin to control epidermal proliferation. Cell 2011, 144:782-795

40. Zhang H, Pasolli HA, Fuchs E: Yes-associated protein (YAP) transcriptional coactivator functions in balancing growth and differentiation in skin. Proc Natl Acad Sci U S A 2011, 108:2270-2275.

41. Tian W, Yu J, Tomchick DR, Pan D, Luo X: Structural and functional analysis of the YAP-binding domain of human TEAD2. Proc Natl Acad SC US A 2010, 107:7293-7298.

42. Chen L, Chan SW, Zhang X, Walsh M, Lim CJ, Hong W: Structural basis of YAP recognition by TEAD4 in the hippo pathway. Genes Dev 2010 24:290-300.

43. Li Z, Zhao B, Wang P, Chen F, Dong Z, Yang H: Structural insights into the YAP and TEAD complex. Genes Dev 2010, 24:235-240.

44. Cao X, Pfaff SL, Gage FH: YAP regulates neural progenitor cell number via the TEA domain transcription factor. Genes Dev 2008, 22:3320-3334.

45. Strano S, Munarriz E, Rossi M, Castagnoli L, Shaul Y, Sacchi A: Physical interaction with Yes-associated protein enhances p73 transcriptional activity. J Biol Chem 2001, 276:15164-15173.

46. Komuro A, Nagai M, Navin NE, Sudol M: WW domain-containing protein YAP associates with ErbB-4 and acts as a co-transcriptional activator for the carboxyl-terminal fragment of ErbB-4 that translocates to the nucleus. J Biol Chem 2003, 278:33334-33341.

47. Yagi $R$, Chen LF, Shigesada K, Murakami Y, Ito Y: A WW domain-containing yes-associated protein (YAP) is a novel transcriptional co-activator. Embo J 1999, 18:2551-2562.

48. Zaidi SK, Sullivan AJ, Medina R, Ito Y, van Wijnen AJ, Stein JL: Tyrosine phosphorylation controls Runx2-mediated subnuclear targeting of YAP to repress transcription. Embo J 2004, 23:790-799.

49. Shao D, Zhai P, Del Re DP, Sciarretta S, Yabuta N, Nojima H: A functional interaction between Hippo-YAP signalling and FoxO1 mediates the oxidative stress response. Nat Commun 2014, 5:3315.

50. Murakami M, Nakagawa M, Olson EN, Nakagawa O: A WW domain protein TAZ is a critical coactivator for TBX5, a transcription factor implicated in Holt-Oram syndrome. Proc Natl Acad Sci U S A 2005, 102:18034-18039.

51. Ferrigno O, Lallemand F, Verrecchia F, L'Hoste S, Camonis J, Atfi A: Yesassociated protein (YAP65) interacts with Smad7 and potentiates its inhibitory activity against TGF-beta/Smad signaling. Oncogene 2002, 21:4879-4884

52. Varelas $X$, Sakuma $R$, Samavarchi-Tehrani P, Peerani R, Rao BM, Dembowy J: TAZ controls Smad nucleocytoplasmic shuttling and regulates human embryonic stem-cell self-renewal. Nat Cell Biol 2008, 10:837-848.

53. Varelas $X$, Samavarchi-Tehrani $P$, Narimatsu M, Weiss A, Cockburn $K$, Larsen BG: The Crumbs complex couples cell density sensing to Hippo-dependent control of the TGF-beta-SMAD pathway. Dev Cell 2010, 19:831-844.
54. Zhao B, Li L, Tumaneng K, Wang CY, Guan KL: A coordinated phosphorylation by Lats and CK1 regulates YAP stability through SCF (beta-TRCP). Genes Dev 2010, 24:72-85.

55. Hao Y, Chun A, Cheung K, Rashidi B, Yang X: Tumor suppressor LATS1 is a negative regulator of oncogene YAP. J Biol Chem 2008, 283:5496-5509.

56. Liu CY, Zha ZY, Zhou X, Zhang H, Huang W, Zhao D: The hippo tumor pathway promotes TAZ degradation by phosphorylating a phosphodegron and recruiting the SCF\{beta\}-TrCP E3 ligase. J Biol Chem 2010, 285:37159-37169.

57. Kim M, Kim M, Lee $S$, Kuninaka S, Saya H, Lee H: cAMP/PKA signalling reinforces the LATS-YAP pathway to fully suppress YAP in response to actin cytoskeletal changes. Embo J 2013, 32:1543-1555.

58. Sudol M, Bork P, Einbond A, Kastury K, Druck T, Negrini M: Characterization of the mammalian YAP (Yes-associated protein) gene and its role in defining a novel protein module, the WW domain. J Bio/ Chem 1995, 270:14733-14741.

59. Imajo M, Miyatake K, limura A, Miyamoto A, Nishida E: A molecular mechanism that links Hippo signalling to the inhibition of Wnt/betacatenin signalling. Embo J 2012, 31:1109-1122.

60. Heallen T, Zhang M, Wang J, Bonilla-Claudio M, Klysik E, Johnson RL: Hippo pathway inhibits Wnt signaling to restrain cardiomyocyte proliferation and heart size. Science 2011, 332:458-461.

61. Xin M, Kim Y, Sutherland LB, Qi X, McAnally J, Schwartz RJ: Regulation of insulin-like growth factor signaling by Yap governs cardiomyocyte proliferation and embryonic heart size. Sci Signal 2011, 4:ra70.

62. Wang J, Park JS, Wei Y, Rajurkar M, Cotton JL, Fan Q: TRIB2 acts downstream of Wnt/TCF in liver cancer cells to regulate YAP and C/EBPalpha function. Mol Cell 2013, 51:211-225.

63. Azzolin L, Zanconato F, Bresolin S, Forcato M, Basso G, Bicciato S: Role of TAZ as mediator of Wnt signaling. Cell 2012, 151:1443-1456.

64. Azzolin L, Panciera T, Soligo S, Enzo E, Bicciato S, Dupont S: YAP/TAZ Incorporation in the beta-Catenin Destruction Complex Orchestrates the Wnt Response. Cell 2014, 158:157-170.

65. Tomlinson V, Gudmundsdottir K, Luong P, Leung KY, Knebel A, Basu S: JNK phosphorylates Yes-associated protein (YAP) to regulate apoptosis. Cell death \& disease 2010, 1:e29.

66. Sun G, Irvine KD: Ajuba family proteins link JNK to Hippo signaling. Sci Signal 2013, 6:ra81.

67. Hamaratoglu F, Willecke M, Kango-Singh M, Nolo R, Hyun E, Tao C: The tumour-suppressor genes NF2/Merlin and Expanded act through Hippo signalling to regulate cell proliferation and apoptosis. Nat Cell Biol 2006, 8:27-36.

68. Badouel C, Gardano L, Amin N, Garg A, Rosenfeld R, Le Bihan T: The FERMdomain protein Expanded regulates Hippo pathway activity via direct interactions with the transcriptional activator Yorkie. Dev Cell 2009, 16:411-420.

69. Oh H, Reddy BV, Irvine KD: Phosphorylation-independent repression of Yorkie in Fat-Hippo signaling. Dev Biol 2009, 335:188-197.

70. Yu J, Zheng Y, Dong J, Klusza S, Deng WM, Pan D: Kibra functions as a tumor suppressor protein that regulates Hippo signaling in conjunction with Merlin and Expanded. Dev Cell 18:288-299.

71. Baumgartner R, Poernbacher I, Buser N, Hafen E, Stocker H: The WW domain protein Kibra acts upstream of Hippo in Drosophila. Dev Cell 2010, 18:309-316.

72. Genevet A, Wehr MC, Brain R, Thompson BJ, Tapon N: Kibra is a regulator of the Salvador/Warts/Hippo signaling network. Dev Cell 2010, 18:300-308.

73. Willecke M, Hamaratoglu F, Kango-Singh M, Udan R, Chen CL, Tao C: The fat cadherin acts through the hippo tumor-suppressor pathway to regulate tissue size. Curr Biol 2006, 16:2090-2100.

74. Silva E, Tsatskis Y, Gardano L, Tapon N, McNeill H: The tumor-suppressor gene fat controls tissue growth upstream of expanded in the hippo signaling pathway. Curr Biol 2006, 16:2081-2089.

75. Bennett FC, Harvey KF: Fat cadherin modulates organ size in Drosophila via the Salvador/Warts/Hippo signaling pathway. Curr Biol 2006, 16:2101-2110.

76. Feng $Y$, Irvine $K D$ : Fat and expanded act in parallel to regulate growth through warts. Proc Natl Acad Sci U S A 2007, 104:20362-20367.

77. Cho E, Feng Y, Rauskolb C, Maitra S, Fehon R, Irvine KD: Delineation of a Fat tumor suppressor pathway. Nat Genet 2006, 38:1142-1150.

78. Rauskolb C, Pan G, Reddy BV, Oh H, Irvine KD: Zyxin links fat signaling to the hippo pathway. PLOS Biol 2011, 9:e1000624.

79. Chen CL, Gajewski KM, Hamaratoglu F, Bossuyt W, Sansores-Garcia L, Tao C The apical-basal cell polarity determinant Crumbs regulates Hippo signaling in Drosophila. Proc Natl Acad Sci U S A 2010, 107:15810-15815. 
80. Ling $C$, Zheng $Y, Y$ in F, Yu J, Huang J, Hong Y: The apical transmembrane protein Crumbs functions as a tumor suppressor that regulates Hippo signaling by binding to Expanded. Proc Natl Acad Sci U S A 2010, 107:10532-10537.

81. Robinson BS, Huang J, Hong Y, Moberg KH: Crumbs regulates Salvador/ Warts/Hippo signaling in Drosophila via the FERM-domain protein Expanded. Curr Biol 2010, 20:582-590.

82. Grzeschik NA, Parsons LM, Allott ML, Harvey KF, Richardson HE: Lgl, aPKC, and Crumbs regulate the Salvador/Warts/Hippo pathway through two distinct mechanisms. Curr Biol 2010, 20:573-581.

83. Poon CL, Lin Jl, Zhang X, Harvey KF: The sterile 20-like kinase Tao-1 controls tissue growth by regulating the Salvador-Warts-Hippo pathway. Dev Cell 2011, 21:896-906.

84. Boggiano JC, Vanderzalm PJ, Fehon RG: Tao-1 phosphorylates Hippo/MST kinases to regulate the Hippo-Salvador-Warts tumor suppressor pathway. Dev Cell 2011, 21:888-895.

85. Wehr MC, Holder MV, Gailite I, Saunders RE, Maile TM, Ciirdaeva E: Saltinducible kinases regulate growth through the Hippo signalling pathway in Drosophila. Nat Cell Biol 2013, 15:61-71.

86. Poon CL, Zhang X, Lin Jl, Manning SA, Harvey KF: Homeodomaininteracting protein kinase regulates Hippo pathway-dependent tissue growth. Curr Biol 2012, 22:1587-1594.

87. Chen J, Verheyen EM: Homeodomain-interacting protein kinase regulates Yorkie activity to promote tissue growth. Curr Biol 2012, 22:1582-1586.

88. Enderle L, McNeill H: Hippo gains weight: added insights and complexity to pathway control. Sci Signal 2013, 6:re7.

89. Irvine KD: Integration of intercellular signaling through the Hippo pathway. Semin Cell Dev Biol 2012, 23:812-817.

90. Staley BK, Irvine KD: Hippo signaling in Drosophila: recent advances and insights. Dev Dyn 2012, 241:3-15

91. Boggiano JC, Fehon RG: Growth control by committee: intercellular junctions, cell polarity, and the cytoskeleton regulate Hippo signaling. Dev Cell 2012, 22:695-702.

92. Grusche FA, Richardson HE, Harvey KF: Upstream regulation of the hippo size control pathway. Curr Biol 2010, 20:R574-R582.

93. Polesello C, Huelsmann S, Brown NH, Tapon N: The Drosophila RASSF homolog antagonizes the hippo pathway. Curr Biol 2006, 16:2459-2465.

94. Praskova M, Khoklatchev A, Ortiz-Vega S, Avruch J: Regulation of the MST1 kinase by autophosphorylation, by the growth inhibitory proteins, RASSF1 and NORE1, and by Ras. Biochem J 2004, 381:453-462.

95. Guo C, Tommasi S, Liu L, Yee JK, Dammann R, Pfeifer GP: RASSF1A is part of a complex similar to the Drosophila Hippo/Salvador/Lats tumorsuppressor network. Curr Biol 2007, 17:700-705.

96. Guo C, Zhang X, Pfeifer GP: The tumor suppressor RASSF1A prevents dephosphorylation of the mammalian STE20-like kinases MST1 and MST2. J Biol Chem 2011, 286:6253-6261.

97. Matallanas D, Romano D, Yee K, Meissl K, Kucerova L, Piazzolla D: RASSF1A elicits apoptosis through an MST2 pathway directing proapoptotic transcription by the p73 tumor suppressor protein. Mol Cell 2007, 27:962-975.

98. Romano D, Matallanas D, Weitsman G, Preisinger C, Ng T, Kolch W: Proapoptotic kinase MST2 coordinates signaling crosstalk between RASSF1A, Raf-1, and Akt. Cancer Res 2010, 70:1195-1203.

99. Zhang N, Bai H, David KK, Dong J, Zheng Y, Cai J: The Merlin/NF2 tumor suppressor functions through the YAP oncoprotein to regulate tissue homeostasis in mammals. Dev Cell 2010, 19:27-38.

100. Benhamouche S, Curto M, Saotome I, Gladden AB, Liu CH, Giovannini M: Nf2/ Merlin controls progenitor homeostasis and tumorigenesis in the liver. Genes Dev 24:1718-1730.

101. Lavado A, He Y, Pare J, Neale G, Olson EN, Giovannini M: Tumor suppressor Nf2 limits expansion of the neural progenitor pool by inhibiting Yap/Taz transcriptional coactivators. Development 2013, 140:3323-3334

102. Li W, You L, Cooper J, Schiavon G, Pepe-Caprio A, Zhou L: Merlin/NF2 suppresses tumorigenesis by inhibiting the E3 ubiquitin ligase CRL4 (DCAF1) in the nucleus. Cell 140:477-490.

103. Kim NG, Koh E, Chen X, Gumbiner BM: E-cadherin mediates contact inhibition of proliferation through Hippo signaling-pathway components. Proc Natl Acad Sci U S A 2011, 108:11930-11935.

104. Genevet A, Tapon N: The Hippo pathway and apico-basal cell polarity. Biochem J 2011, 436:213-224
105. Yu FX, Zhao B, Panupinthu N, Jewell JL, Lian I, Wang LH: Regulation of the Hippo-YAP pathway by G-protein-coupled receptor signaling. Cell 2012, 150:780-791.

106. Mo JS, Yu FX, Gong R, Brown JH, Guan KL: Regulation of the Hippo-YAP pathway by protease-activated receptors (PARs). Genes Dev 2012, 26:2138-2143.

107. Miller E, Yang J, DeRan M, Wu C, Su Al, Bonamy GM: Identification of serum-derived sphingosine-1-phosphate as a small molecule regulator of YAP. Chem Biol 2012, 19:955-962

108. Yu FX, Zhang Y, Park HW, Jewell JL, Chen Q, Deng Y: Protein kinase A activates the Hippo pathway to modulate cell proliferation and differentiation. Genes Dev 2013, 27:1223-1232.

109. Fan R, Kim NG, Gumbiner BM: Regulation of Hippo pathway by mitogenic growth factors via phosphoinositide 3-kinase and phosphoinositidedependent kinase-1. Proc Natl Acad Sci U S A 2013, 110:2569-2574.

110. Reddy BV, Irvine KD: Regulation of Hippo signaling by EGFR-MAPK signaling through Ajuba family proteins. Dev Cell 2013, 24:459-471.

111. Dupont S, Morsut L, Aragona M, Enzo E, Giulitti S, Cordenonsi M: Role of YAP/TAZ in mechanotransduction. Nature 2011, 474:179-183.

112. Aragona M, Panciera T, Manfrin A, Giulitti S, Michielin F, Elvassore N: A mechanical checkpoint controls multicellular growth through YAP/ TAZ regulation by actin-processing factors. Cell 2013, 154:1047-1059.

113. Brade T, Pane LS, Moretti A, Chien KR, Laugwitz KL: Embryonic heart progenitors and cardiogenesis. Cold Spring Harbor perspectives in Med 2013, 3:a013847.

114. Bruneau BG, Nemer G, Schmitt JP, Charron F, Robitaille L, Caron S: A murine model of Holt-Oram syndrome defines roles of the T-box transcription factor Tbx5 in cardiogenesis and disease. Cell 2001, 106:709-721.

115. von Gise A, Lin Z, Schlegelmilch K, Honor LB, Pan GM, Buck JN: YAP1, the nuclear target of Hippo signaling, stimulates heart growth through cardiomyocyte proliferation but not hypertrophy. Proc Natl Acad Sci U S A 2012, 109:2394-2399.

116. Bergmann O, Bhardwaj RD, Bernard S, Zdunek S, Barnabe-Heider F, Walsh S. Evidence for cardiomyocyte renewal in humans. Science 2009, 324:98-102.

117. Senyo SE, Steinhauser ML, Pizzimenti CL, Yang VK, Cai L, Wang M: Mammalian heart renewal by pre-existing cardiomyocytes. Nature 2013, 493:433-436.

118. Poss KD, Wilson LG, Keating MT: Heart regeneration in zebrafish. Science 2002, 298:2188-2190.

119. Lian I, Kim J, Okazawa H, Zhao J, Zhao B, Yu J: The role of YAP transcription coactivator in regulating stem cell self-renewal and differentiation. Genes Dev 2010, 24:1106-1118.

120. Judson RN, Tremblay AM, Knopp P, White RB, Urcia R, De Bari C: The Hippo pathway member Yap plays a key role in influencing fate decisions in muscle satellite cells. J Cell Sci 2012, 125:6009-6019.

121. Yimlamai D, Christodoulou C, Galli GG, Yanger K, Pepe-Mooney B, Gurung B, Shrestha K, Cahan P, Stanger BZ, Camargo FD: Hippo pathway activity influences liver cell fate. Cell 2014, 157:1324-1338.

122. Del Re DP, Yang Y, Nakano N, Cho J, Zhai P, Yamamoto T: Yes-associated protein isoform 1 (Yap1) promotes cardiomyocyte survival and growth to protect against myocardial ischemic injury. J Biol Chem 2013, 288:3977-3988

123. Xin M, Kim Y, Sutherland LB, Murakami M, Qi X, McAnally J: Hippo pathway effector Yap promotes cardiac regeneration. Proc Natl Acad Sci U S A 2013, 110:13839-13844.

124. Lin Z, von Gise A, Zhou P, Gu F, Ma Q, Jiang J, Yau AL, Buck JN, Gouin KA, van Gorp PR, Zhou B, Chen J, Seidman JG, Wang DZ, Pu WT: CardiacSpecific YAP Activation Improves Cardiac Function and Survival in an Experimental Murine Myocardial Infarction Model. Circ Res 2014.

125. Heallen T, Morikawa Y, Leach J, Tao G, Willerson JT, Johnson RL: Hippo signaling impedes adult heart regeneration. Development 2013, 140:4683-4690.

126. Porrello ER, Mahmoud Al, Simpson E, Hill JA, Richardson JA, Olson EN: Transient regenerative potential of the neonatal mouse heart. Science 2011, 331:1078-1080.

127. Creasy $C L$, Chernoff J: Cloning and characterization of a human protein kinase with homology to Ste20. J Biol Chem 1995, 270:21695-21700.

128. Graves JD, Gotoh Y, Draves KE, Ambrose D, Han DK, Wright M: Caspasemediated activation and induction of apoptosis by the mammalian Ste20-like kinase Mst1. Embo J 1998, 17:2224-2234. 
129. Yamamoto S, Yang G, Zablocki D, Liu J, Hong C, Kim SJ: Activation of Mst1 causes dilated cardiomyopathy by stimulating apoptosis without compensatory ventricular myocyte hypertrophy. J Clin Invest 2003, 111:1463-1474.

130. Odashima M, Usui S, Takagi H, Hong C, Liu J, Yokota M: Inhibition of endogenous Mst1 prevents apoptosis and cardiac dysfunction without affecting cardiac hypertrophy after myocardial infarction. Circ Res 2007, 100:1344-1352.

131. Nishida K, Kyoi S, Yamaguchi O, Sadoshima J, Otsu K: The role of autophagy in the heart. Cell Death Differ 2009, 16:31-38.

132. Maejima Y, Kyoi S, Zhai P, Liu T, Li H, Ivessa A: Mst1 inhibits autophagy by promoting the interaction between Beclin1 and Bcl-2. Nat Med 2013, 19:1478-1488.

133. Del Re DP, Matsuda T, Zhai P, Maejima Y, Jain MR, Liu T: Mst1 promotes cardiac myocyte apoptosis through phosphorylation and inhibition of Bcl-xL. Mol Cell 2014, 54:639-650.

134. Ardestani A, Paroni F, Azizi Z, Kaur S, Khobragade V, Yuan T: MST1 is a key regulator of beta cell apoptosis and dysfunction in diabetes. Nat Med 2014, 20:385-397.

135. Matsui Y, Nakano N, Shao D, Gao S, Luo W, Hong C: Lats2 Is a Negative Regulator of Myocyte Size in the Heart. Circ Res 2008, 103:1309-1318.

136. Oceandy D, Pickard A, Prehar S, Zi M, Mohamed TM, Stanley PJ: Tumor suppressor Ras-association domain family 1 isoform $A$ is a novel regulator of cardiac hypertrophy. Circulation 2009, 120:607-616.

137. Del Re DP, Matsuda T, Zhai P, Gao S, Clark GJ, Van Der Weyden L. Proapoptotic Rassf1A/Mst1 signaling in cardiac fibroblasts is protective against pressure overload in mice. $J$ Clin Invest 2010, 120:3555-3567.

138. Overholtzer M, Zhang J, Smolen GA, Muir B, Li W, Sgroi DC: Transforming properties of YAP, a candidate oncogene on the chromosome 11q22 amplicon. Proc Natl Acad Sci U S A 2006, 103:12405-12410.

139. Steinhardt AA, Gayyed MF, Klein AP, Dong J, Maitra A, Pan D: Expression of Yes-associated protein in common solid tumors. Hum Pathol 2008, 39:1582-1589

140. Cai J, Zhang N, Zheng Y, de Wilde RF, Maitra A, Pan D: The Hippo signaling pathway restricts the oncogenic potential of an intestinal regeneration program. Genes Dev 2010, 24:2383-2388.

141. Lee KP, Lee JH, Kim TS, Kim TH, Park HD, Byun JS: The Hippo-Salvador pathway restrains hepatic oval cell proliferation, liver size, and liver tumorigenesis. Proc Natl Acad Sci U S A 2010, 107:8248-8253.

142. Zhang X, George J, Deb S, Degoutin JL, Takano EA, Fox SB: The Hippo pathway transcriptional co-activator, YAP, is an ovarian cancer oncogene. Oncogene 2011, 30:2810-2822.

143. Zhou D, Zhang Y, Wu H, Barry E, Yin Y, Lawrence E: Mst1 and Mst2 protein kinases restrain intestinal stem cell proliferation and colonic tumorigenesis by inhibition of Yes-associated protein (Yap) overabundance. Proc Natl Acad Sci U S A 2011, 108:E1312-E1320.

144. Camargo FD, Gokhale S, Johnnidis JB, Fu D, Bell GW, Jaenisch R: YAP1 increases organ size and expands undifferentiated progenitor cells. Curr Biol 2007, 17:2054-2060.

145. Chen Z, Friedrich GA, Soriano P: Transcriptional enhancer factor 1 disruption by a retroviral gene trap leads to heart defects and embryonic lethality in mice. Genes Dev 1994, 8:2293-2301.

146. Morin-Kensicki EM, Boone BN, Howell M, Stonebraker JR, Teed J, Alb JG: Defects in yolk sac vasculogenesis, chorioallantoic fusion, and embryonic axis elongation in mice with targeted disruption of Yap65. Mol Cell Biol 2006, 26:77-87.

147. van Berlo JH, Kanisicak O, Maillet M, Vagnozzi RJ, Karch J, Lin SC: c-kit+ cells minimally contribute cardiomyocytes to the heart. Nature 2014, 509:337-341.

doi:10.1186/s40169-014-0027-0

Cite this article as: Del Re: The hippo signaling pathway: implications for heart regeneration and disease. Clinical and Translational Medicine 2014 3:27.

\section{Submit your manuscript to a SpringerOpen ${ }^{\odot}$ journal and benefit from:}

- Convenient online submission

- Rigorous peer review

- Immediate publication on acceptance

- Open access: articles freely available online

- High visibility within the field

- Retaining the copyright to your article 